\title{
DEVELOPMENT OF TELEMEDICINE SYSTEM
}

\author{
Denis Kuznetsov ${ }^{\mathrm{a}}$ \\ National Research Tomsk State University, 634050, Tomsk, Russia
}

\begin{abstract}
The tendency of computer technologies implementation to all areas of life is obvious and indisputable. Informatization of such area as medicine, gives huge advantage to health care service. For example, use of telemedicine technologies will allow making professional medical care to the removed patient. The telemedicine is distance medical care of patients by means of computers, the Internet and other communication technologies. Nowadays, telemedicine is developed subarea of health services delivery and medical education system. It is the reliable and available instrument for the improvement of healthcare systems. In the remote regions of Russia forming of medical services is rather difficult, but last years, Russian organizations show interest in implementation of telemedical services. Telemedicine promotes cooperation between the medical organizations, allows organizing effective system of medical personnel training. Priority tasks of telemedicine development are following: standardization of the applied medical, computer and telecommunication technologies, development of information resources. Approach "telemedicine" is rather new, especially for Russia, so the problem of healthcare services improvement is a priority task of our society and one of the main aspects in policy of the Russian Federation. In this paper, we raise the issue of algorithms development for carrying out the medical consultations in telemedicine system
\end{abstract}

\section{Introduction}

All types of information: official, confidential and descriptive - are stored in the database (D), with which the system of telemedical consultations needs to interact. The module of the database provides interpretation of authorized user requests, with next transmission to database, data acceptance, encoding and interpretation of results $[1,2]$. The module of the database interacts with the desktop module, through safety system, receiving from it requests of users. Also, it exchanges data with bases of all system via the module of network interaction which is responsible for authorization of users, encoding and protection of confidential information in a D.

\footnotetext{
${ }^{a}$ Corresponding author: novorostomsk@gmail.com
} 


\section{Database Module}

Functions of the D module, according to solvable tasks, are divided into two classes: for operation with the database and for dataful operation. The first are necessary for link establishment with PostGreSQL, for transmissions of requests, acceptances of responses. The second - for provision of data to other system modules in a necessary look and a format [4].

Figure 1 gives the algorithm of $\mathrm{D}$ module operation. Connection setup with database happens at the program start stage during initialization of system resources. The Delphi component, which is used for operation with different databases - ADOConnection of TADOConnection classes, is created. After component creation, initialization string assigns to the component, also other necessary properties are set and, at last, connection opens. Further, initialization string involves authentication and service data, which is necessary for connection with the database. If connection with a $\mathrm{D}$ was not set for any reason, the module under abnormal condition finishes a program runtime [5].

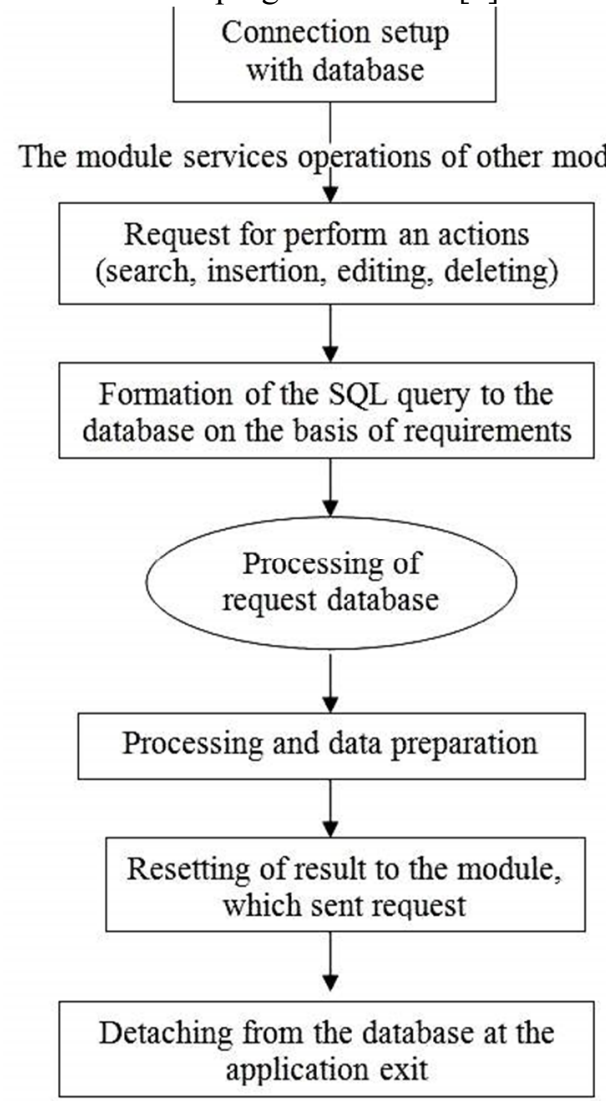

Figure 1. Schematic image of algorithm of operation of AIS TMC databasemodule.

\section{Execution of SQL queries}

Execution of SQL queries to the database is carried out by means of ADOQuery delphicomponent of TADOQuery class. Depending on type of request there are different functions of their execution. As follows, the SQL requests, implying availability of the answer from the database in the form of any data (requests like SELECT). Requests are 
carried out by function, which returns the ADOQuery component as result of the accomplishment, allowing to conduct, further work with the data obtained from a D. On the contrary, requests as INSERT, UPDATE, DELETE do not imply return from a D of any data (except confirmation about successful accomplishment of request) therefore the ADOQuery component is destroyed after accomplishment of function in order to avoid memory leakages $[6,7]$.

Upon the end of operation of application, the ADOConnection component finishes connection with a $\mathrm{D}$, and then is destroyed.

Both components: ADOConnection and ADOQuery - are a part of the ADODB module of the Delphi CodeGear 2009 language.

In order that the AIS TMC system interacted with D in unitary way, we use the mechanism of automatic formation of SQL queries to D. Special object - Delphi class was developed. Object ensures functioning of basic functions with the database - TDB Unit or "Database object". All information units of system: users, patients, teleconsultations, etc. are based on this object [9].

The database object is the central class for all elements of AIS TMC system. So, we will describe hierarchical structure of D objects.

The class TWorkUnit is a main base class. Immediate ancestor of all executive classes of objects descriptions system:

- TWUTMUsers (class of users);

- TWU_TM_GroupsUsers (class of users groups);

- TWUTMPatients (class of patients).

For example, users of AIS TMC have different characteristics: login, password, identification data. Information on these characteristics and their description are set in TWU TM Users class, for patients - respectively in the TWU TM Patients class, etc. Copies of these classes are the working tool of system. They service data and interactions, store information, control a status of a D and the tables [8].

\section{Schematically hierarchy of D objects}

All derived classes of TWorkUnit, for example TWU TM Users, use the universal algorithms of TWorkUnit class, implement generation of SQL queries to the database for formation of tables, selection and data modification in a $\mathrm{D}$, based on structure description of objects, defined in derived classes. They also implement data synchronization, data transformation, formation of the interface for dataful operation, activation of encoding and decoding functions.The executive classes of descriptions are extensions of TWorkUnit class, which contain all information about objects.

In turn, TWorkUnit class comprises a subobject - the TDB Unit class in which all tools for work with SQL inquiries and data are concentrated. Figure 2 presents schematically hierarchy of classes [10].

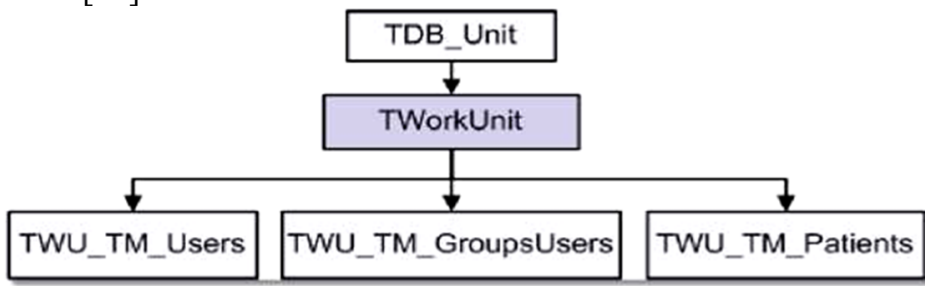

Figure 2. Schematically hierarchy of D objects of AIS TMC.

Objects of TWUTMUsers, TWUTMGroupsUsers, etc. are representation of entities of users of AIS TMC, patients, etc in Delphi (program implementation of objects). Copies of 
TWU TM Users class in the AIS TMC system formally correspond to patients (in other words, each patient is a copy of derived class (patients with parent class the Homo sapiens). Thus, real patients (as well as other information units, for example, users or groups) correspond in the program to copies of the TWU TM Users class which, in turn as a data set correspond to record set in D tables.

All derived classes of TWorkUnit work by the general principle.Iin Delphi selected variable corresponds to each characteristic of a class, in the database - a field or a set of fields of the table. The characteristic has the data format (number, a line, a set of values, the image, etc.), and also a role which defines semantic loading of the characteristic. For example, the surname of the practicing doctor - the user of AIS TMC - is important only for other users of system, and login and the indicator of this doctor - key characteristics, they are processed essentially differently [12].

The description of object is created from characteristics. Based on this description, TDB Unit creates SQL queries to the database. In case of initialization of derived classes copies of TWorkUnit class, function of check of D integrity checking and creation of tables is executed. Figure 3 provides algorithm of functioning.

At a stage of system parameters preparation we select resources under auxiliary variable and set primary value. Then we begin search of the tables, serviced by this class. If the table doesn't exist - we start SQL query formation by table creation [11]. Otherwise check begins whether all fields of the table corresponding to attributes are present at it. Thus, the database always is in a status necessary for us, and database changes dynamically are traced and improve, and we exclude situations when the SQL query to this table comes to the end with an error because of its incorrect structure.

We creat SELECT section of SQL query in the same way. SELECT section is used for data sampling.

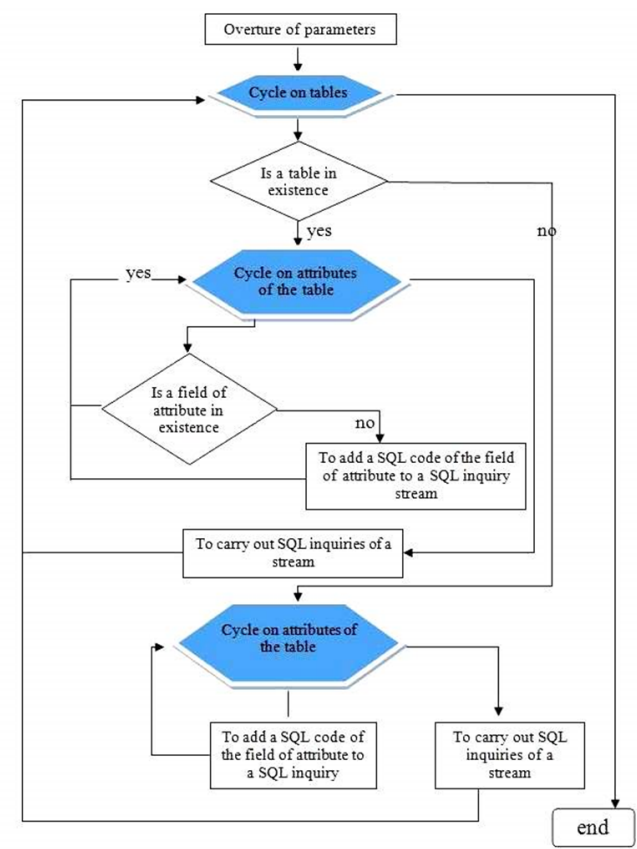

Figure 3. Control-flow chart of D table control.

Formally SELECT section of SQL query looks as follows: "SELECT list of the FROM fields list of the tables WHERE conditions".

The system collects each section in turn, based on characteristics of attributes, - each 
attribute belongs to the table, possesses a certain data type, and also can be connected somehow to other attribute from other table. In addition, during the search we specified additional conditions which need to be considered [3].

The system automatically creates request.

Schematically the algorithm is provided in Figure 4.

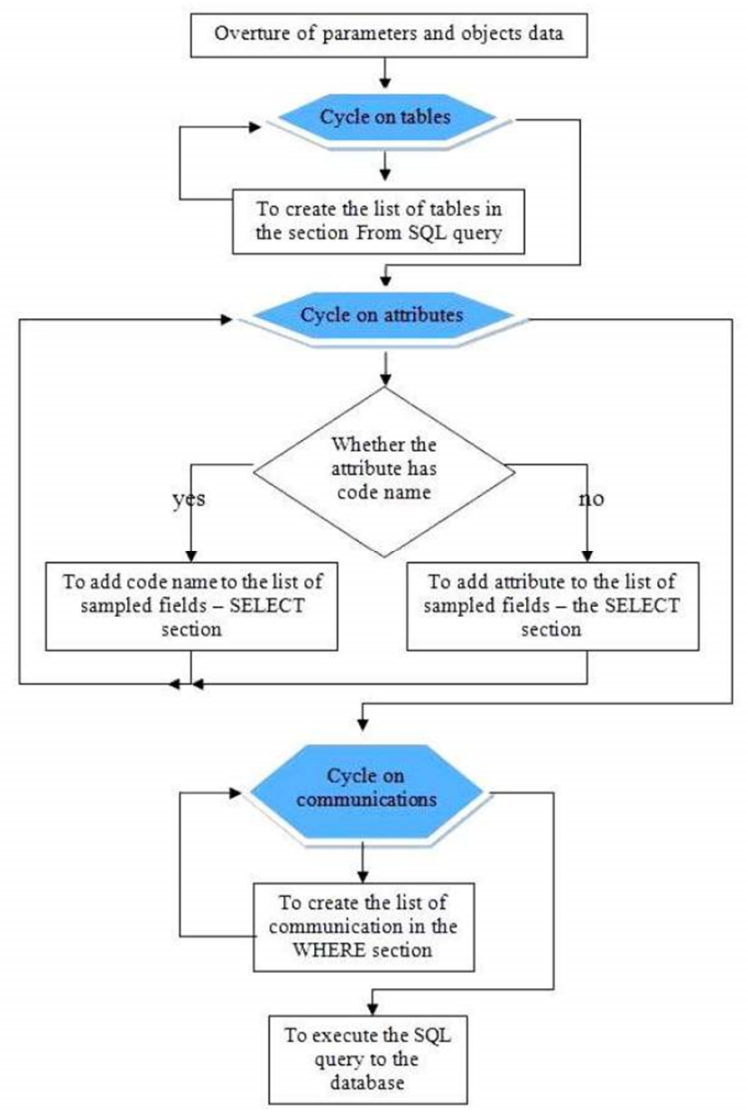

Figure 4. Control-flow chart of SELECT section formation.

For a data recording in the database the system also creates the SQL query depending on the task, the INSERT or UPDATE type.

During the recording, the copy of the "object of Delphi" class contains data, which are required to be added to basis. As incremental fields of one tables can be keys at others, for tables requests of an insertion are created and executed sequentially. Thus, attributes of each table get over [12].

At this time system, leaning on a role and sometimes on type, defines how data are stored and how to add them to request, necessity of encoding them, etc. In completion,we executed SQL queryfor each table, result of which is the indicator of new record.

Figure 5 presents flowchart of this algorithm. 


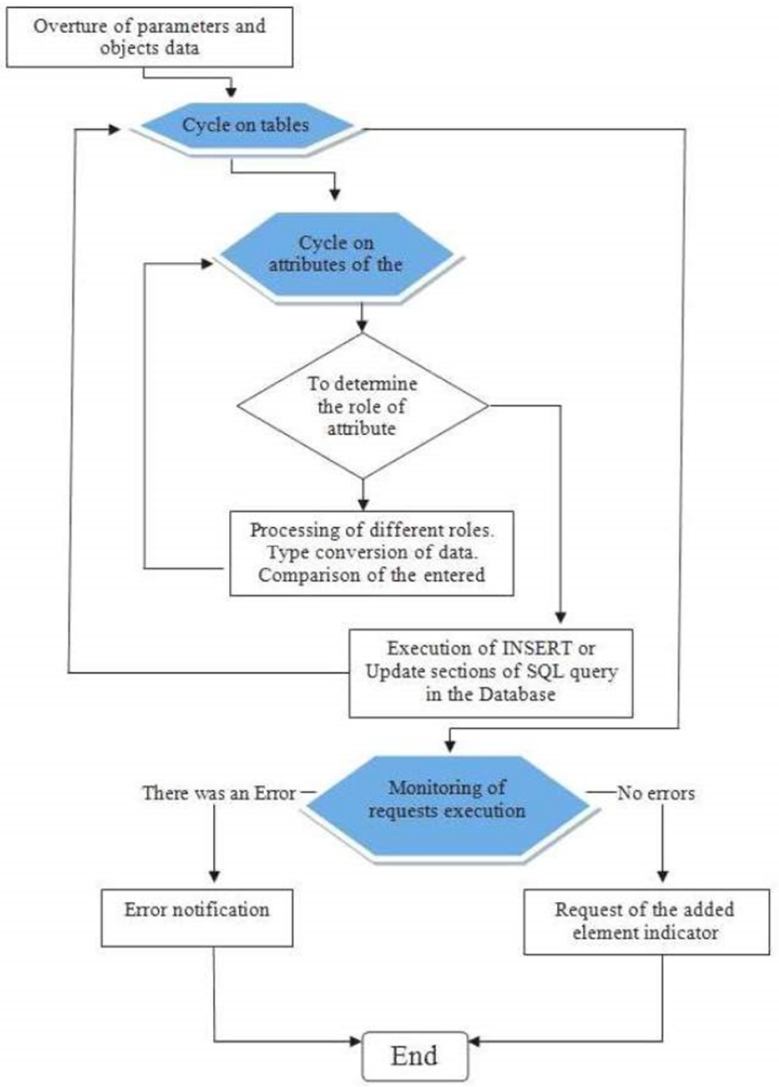

Figure 5. Control-flow chart of insert and editing records in D.

\section{Conclusion}

Thus, the system itself controls processes of operation with the database independently, provides the flexibility in construction and changing of D structure, which allows for developer quicker program creation. For program formation we developed different modules: the module of network interaction, module of security policy, the module of an exchange of video information, the module of control demonstration tasks, the module of desktop, but it will be stated in the following materials.

The developed telemedical system integrates all types of healthcare institutions - the central and regional regulations, the central, regional and region clinics and hospitals, medical academies and institutes, archives and libraries and other treatment-andprophylactic establishments.

\section{Acknowledgements}

The paper was written as part of the research project No. 8.2.31.2015, carried out with the support of the Program "Research Foundation of Tomsk State University named after D.I. Mendeleev" in 2015 - 2016. 


\section{References}

[1] D.A. Person, J.S. Hedson, K.J. Gunawardane, Telemedicine Journal and e-Health 9, 95 (2003) doi: 10.1089/153056203321593642

[2] A. Szot et al., International Journal of Medical Informatics 73, 65 (2004) doi: 10.1016/j.ijmedinf.2003.10.002

[3] V. Patterson et al., Journal of Telemedicine and Telecare 7, 52(2001) doi: 10.1258/1357633011936714

[4] I. Qaddoumi et al., Pediatric Blood \& Cancer 48, 39 (2007) doi: $10.1002 / \mathrm{pbc} .21085$

[5] P.V. Pradeep et al.,World Journal of Surgery 31, 1665 (2007) doi: $10.1007 / \mathrm{s} 00268-007-9108-1$

[6] D. N. Kuznetsov, V. I. Syryamkin, NEW OPERATIONAL TECHNOLOGIES (NEWOT'2015) 1688, 040004 (2015) doi:10.1063/1.4936037

[7] E.M. Strehle, N. Shabde, Archives of Disease in Childhood 91, 956(2006)

[8] I Matveev, E Siemens, A Yurchenko and D Kuznetsov, IOP Conference Series: Materials Science and Engineering 132, 012010 (2016) doi: 10.1088/1757$899 X / 132 / 1 / 012010$

[9] WHO Group Consultation on Health Telematics, A health telematics policy in support of WHO's Health-for-all strategy for global health development : report of the WHO Group Consultation on Health Telematics (World Health Organization, Geneva,1997)

[10] D. N. Kuznetsov, V. I. Syryamkin, Innovation theory-2015, 453 (2015)

[11] W. Einthoven, Archives Internationales de Physiologie (4), 132 (1906)

[12] D. N. Kuznetsov, V. I. Syryamkin, Telecommunications (8), 44(2015) 